\title{
Perfil das internações de crianças por malformações congênitas do aparelho circulatório no Brasil de 2010 a 2020
}

\author{
Profile of Children's hospitalization due to congenital malformations of the circulatory \\ apparatus in Brazil from 2010 to 2020
}

\author{
Laís Fernanda Duarte Sampaio ${ }^{1 *}$, Nilo Manoel Pereira Vieira Barreto², Helena França Correia ${ }^{3}$ \\ ${ }^{1}$ Fisioterapeuta. Mestranda do Programa de Pós-graduação Processos Interativos dos Órgãos e Sistemas. \\ Universidade Federal da Bahia - UFBA; ${ }^{2}$ Enfermeiro. Mestre em Processos Interativos dos Órgãos e Sistemas - \\ UFBA; ${ }^{3}$ Fisioterapeuta. Doutora em Medicina e Saúde Humana. Professora associada. Programa de Pós-graduação \\ em Processos Interativos de Órgãos e Sistemas. Instituto de Ciências da Saúde - UFBA
}

\begin{abstract}
Resumo
Introdução: a malformação congênita do aparelho circulatório é definida como qualquer anormalidade na estrutura e/ou função do coração durante a fase do desenvolvimento embrionário, podendo interferir no fluxo sanguíneo. Suas manifestações ocorrem em qualquer fase do desenvolvimento. No Brasil, essa enfermidade representa a segunda principal causa de mortalidade em crianças menores de um ano, caracterizando-se um problema de saúde pública. Objetivo: descrever o perfil das internações de crianças por malformações congênitas do aparelho circulatório no Brasil, de 2010 a 2020. Metodologia: trata-se de um estudo ecológico, realizado por meio de dados secundários, no período de 2010 a 2020. Analisaram-se as variáveis número de internações, sexo, região, raça/cor, faixa etária, custos hospitalares, óbito e taxa de mortalidade. As informações coletadas foram armazenadas e analisadas no software Microsoft Excel Office, versão de 365. Resultados: entre 2010 e 2020, foram notificadas 118.792 internações por essa comorbidades de crianças por malformação congênita do aparelho circulatório, sendo $51 \%$ do sexo masculino, com predomínio da cor branca $(36 \%)$, tendo a região Sudeste apresentado o maior percentual de internações $(43,5 \%)$. Observou-se maior ocorrência em crianças abaixo de um ano (62,7\%), gerando um custo de 650 milhões de reais em serviços hospitalares. O maior percentual de internações ocorreu em 2019 (10,9\%). Houve 10.477 óbitos entre 2010 e 2020 (8,8\%), sendo 85,8\% na população abaixo de um ano e 37,7\% na região Sudeste. A taxa de mortalidade de crianças por essa comorbidade foi de 8,82/100 mil habitantes. Conclusão: observou-se que as malformações congênitas do aparelho circulatório, no período analisado, apresentaram uma distribuição homogênea em relação ao sexo, sendo mais frequentes na raça/cor branca, na região Sudeste e na faixa etária menor que um ano, gerando altos custos em serviços hospitalares.

Palavras-chave: Anormalidades congênitas. Epidemiologia. Mortalidade.
\end{abstract}

\begin{abstract}
Introduction: congenital malformation of the circulatory system is defined as any abnormality in the structure and/or function of the heart during the embryonic development phase, which may interfere with blood flow. Its manifestations can occur at any stage of development. In Brazil, it represents the second leading cause of mortality in children under one year of age, characterizing a public health problem. Objective: to describe the profile of hospitalizations of children due to congenital malformations of the circulatory system in Brazil, from 2010 to 2020. Method: this is an ecological study, using secondary data from the Informatics Department of the Unified Health System, by the Hospital Information System of the Unified Health System, from 2010 to 2020. The following variables were analyzed: number of admissions, sex, region, race/color, age group, hospital costs, death and mortality rate. From the information acquired by the TabNet tool, for purposes of processing and analyzing the information collected, data were stored and analyzed in Microsoft Excel Office software, version 365. Results: between 2010 and 2020, 118,792 hospitalizations were reported in children due to congenital malformation of the circulatory system, $51 \%$ of whom were male, with a predominance of whites (36\%). Southeast region was the region with the highest percentage of hospitalizations (43.5\%). A higher occurrence was observed in children under one year of age (62.7\%), generating a cost of 650 million reais in hospital services. The highest percentage of hospitalizations was registered in the year 2019 (10.9\%). There were 10,477 deaths between 2010 and 2020 (8.8\%), being 85.8\% in the population under one year of age and $37.7 \%$ in the Southeast region. The mortality rate of children due to this comorbidity was $8.82 / 100$ thousand inhabitants. Conclusion: it was observed that the congenital malformations of the circulatory system, in the analyzed period, presented a homogeneous distribution in relation to sex, being more frequent in the white race/color, in the Southeast region and in the age group under one year, generating high costs in services hospitals. Keywords: Congenital Abnormalities. Epidemiology. Mortality.
\end{abstract}

Correspondência/Corresponding: *Laís Fernanda Duarte Sampaio - Instituto de Ciências da Saúde - UFBA - End: Av. Reitor Calmon, s/n - Vale do Canela - 40.110-902 Salvador - BA - Tel.: (71) 3283-8900 - E-mail: laisduarte.fisio@hotmail.com

\section{INTRODUÇÃO}

As malformações congênitas (MC) são definidas como qualquer defeito estrutural e/ou funcional presente em um indivíduo desde o nascimento, com complicações que podem se manifestar ao longo da vida, trazendo impacto 
no crescimento e no desenvolvimento infantil (SOARES, 2020). Essas repercussões geram uma alta demanda nos serviços de saúde, necessitando de uma atenção especializada multiprofissional e interdisciplinar, tornando-se um problema de saúde pública. (DUTRA; LIMA; PINTO JUNIOR, 2017; RODRIGUES et al., 2014).

Dentre as MC, as do aparelho circulatório constituem a principal causa de internações no Brasil. É a segunda principal causa de mortalidade em crianças menores de um ano e a terceira causa de óbito até os 30 dias de vida no Brasil (BRASIL. MINISTÉRIO DA SAÚDE, 2017). Esses dados podem ser justificados pela escassez de um diagnóstico precoce, resultando em tratamentos inefetivos ou tardios, dificultando um melhor prognóstico (MIYAGUE et al., 2003).

Alguns fatores de risco tendem a aumentar a incidência da malformação congênita do aparelho circulatório (MAC). Entre eles, o histórico familiar, principalmente entre parentes de primeiro grau, fatores maternos, tais como as doenças crônicas, como a diabetes ou a fenilcetonúria mal controladas, consumo de álcool, exposição a toxinas ambientais e infecções (HOCKENBERRY, 2011).

Aproximadamente um quarto dos recém-nascidos (RN) com MAC podem apresentar sinais já no primeiro ano de vida (MARI, 2015). Os mais clássicos são as dificuldades e o cansaço ou a cianose durante a amamentação, casos em que pode ser necessária intervenção cirúrgica imediata. Além disso, hospitalizações recorrentes e maior risco de complicações podem ocorrer e evoluir a óbito no primeiro ano de vida (SANTOS et al., 2012). Portanto, o diagnóstico precoce torna-se necessário, podendo ser realizado ainda de forma intrauterina, através do ecocardiograma fetal (RICHMOND; WREN, 2001).

As MAC impactam diretamente na mortalidade infantil e dependem de diversos fatores, tais quais a prevalência, a qualidade e a disponibilidade dos serviços de saúde-hospitalares/cirúrgicos - e requerem medidas da atenção primária à saúde (LEITE; MIZIARA; VELOSO 2010). Portanto, conhecê-las e identificar os principais fatores de risco permite melhores intervenções precoces, repercutindo positivamente na qualidade de vida das crianças/ famílias acometidas. Além disso, tendo em vista que o diagnóstico tardio repercute em um pior prognóstico, torna-se necessário identificar o perfil epidemiológico das crianças com MAC, favorecendo o planejamento das ações de promoção de saúde e prevenção de doenças, contribuindo para redução da mortalidade infantil. Ante o exposto, o presente estudo teve como objetivo descrever o perfil das internações de crianças por MAC no Brasil, entre o período compreendido de 2010 a 2020.

\section{METODOLOGIA}

Trata-se de um estudo epidemiológico, ecológico e descritivo, desenvolvido a partir de dados secundários do Departamento de Informática do Sistema Único de Saúde (DATASUS) e pelo Sistema de Informações Hospitalares do Sistema único de Saúde (SIH/SUS), utilizando-se a ferramenta Informações em Saúde (TabNet).

A busca dos dados foi realizada no mês de julho de 2021, com uma amostra constituída de crianças nascidas vivas residentes no Brasil, com 10 anos incompletos, seguindo a classificação adotada pela Organização Mundial de Saúde (OMS) com diagnóstico de MAC (CID 10 Q24). As variáveis foram analisadas de acordo a macrorregião geoeconômica, sendo elas: número de internação, sexo (masculino e feminino), região (Região Norte, Nordeste, Sudeste, Sul e Centro-Oeste), raça/cor (branca, preta, parda, amarela, indígena e sem informação), faixa etária (menor que 1 ano, de 1 a 4 anos, de 5 a 9 anos), custos hospitalares, óbitos e taxa de mortalidade (a cada 100 mil habitantes), considerando o período compreendido entre janeiro de 2010 a dezembro 2020.

Com base nas informações obtidas pela ferramenta TabNet, para fins de processamento e análise das informações coletadas, os dados foram armazenados e analisados no software Microsoft Excel Office, versão de 365. A pesquisa não apresenta implicações éticas devido à utilização de dados secundários de domínio público, não tendo sido necessário submissão ao Comitê de Ética em Pesquisa.

\section{RESULTADOS}

Entre os anos de 2010 e 2020, foram notificados um total de 118.792 internações por malformações do aparelho circulatório em crianças até 9 anos de idade, com pouca variância entre os anos analisados, sendo possível notar um aumento entre o ano de 2010 (8,17\%; 9706/ 118792) e 2019 (10,94\%; 13003/118792). Entretanto, houve uma queda das notificações em 2020 (9,99\%; 1868/118792) (Figura 1).

Figura 1 - Internações por malformações congênitas do aparelho circulatório por ano de processamento e sexo

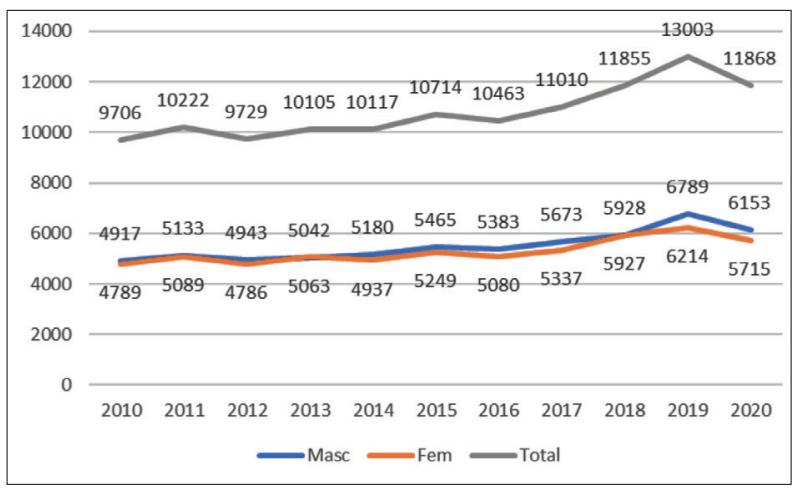

Fonte: Elaborado com base nos dados do Datasus (2021)

Dentre as internações, 51\% (60606) foram do sexo masculino. Houve predomínio da cor branca seguida da parda, com 35,99\% (42756) e $28,46 \%$ (33819), respectivamente. Contudo, um total de 33,15\% (39381/118792) não informou a cor da pele. Quanto à idade, um total de 
62,77\% (74.561) das internações por MAC ocorreram na população abaixo de um ano, sendo menores nas faixas etárias de 1 a 4 anos $(25,34 \% ; 30100)$ e 5 a 9 anos (11,89\%; 14131). O Sudeste seguido do Nordeste foram as regiões que apresentaram os maiores percentuais de internações, com $43,45 \%$ (51621) e 25,63\% (30447), respectivamente (Tabela 1).

Tabela 1 - Descrição das internações hospitalares de crianças com malformações congênitas do aparelho circulatório para as variáveis de cor, faixa etária e região de internação, segundo o sexo, de 2010 a 2020.

\begin{tabular}{lrrr}
\hline & \multicolumn{2}{c}{ Sexo } & Total \\
\cline { 2 - 3 } Variáveis & Masculino & Feminino & n (\%) \\
\cline { 2 - 3 } & $\mathbf{n}(\%)$ & $\mathbf{n}(\%)$ & \\
\hline Raça/cor & & & \\
Branco & $21692(35,79 \%)$ & $21064(36,20 \%)$ & $42756(35,99 \%)$ \\
Pardo & $17433(28,76 \%)$ & $16386(28,16 \%)$ & $33819(28,47 \%)$ \\
Preto & $1028(1,70 \%)$ & $1069(1,83 \%)$ & $2097(1,76 \%)$ \\
Amarela & $237(0,39 \%)$ & $227(0,39 \%)$ & $464(3,90 \%)$ \\
Indígena & $125(0,21 \%)$ & $150(0,26 \%)$ & $275(0,23 \%)$ \\
Não declarado & $20091(33,15 \%)$ & $19290(33,16 \%)$ & $39381(29,65 \%)$ \\
Faixa etária & & & \\
< 1 ano & $38773(63,97 \%)$ & $35788(61,50 \%)$ & $74561(62,77 \%)$ \\
1 a 4 anos & $14853(24,50 \%)$ & $15247(26,20 \%)$ & $30100(25,34 \%)$ \\
5 a 9 anos & $6980(11,53 \%)$ & $7151(12,30 \%)$ & $14131(11,89 \%)$ \\
Regiões de internação & & & \\
Sudeste & $26296(43,39 \%)$ & $25325(43,52 \%)$ & $51621(43,45 \%)$ \\
Nordeste & $15644(25,81 \%)$ & $14803(25,44 \%)$ & $30447(25,63 \%)$ \\
Sul & $9661(15,94 \%)$ & $9101(15,64 \%)$ & $18762(15,79 \%)$ \\
Norte & $4507(7,44 \%)$ & $4564(7,85 \%)$ & $9071(7,64 \%)$ \\
Centro-Oeste & $4498(7,42 \%)$ & $4393(7,55 \%)$ & $8891(7,49 \%)$ \\
Total & $60606(100 \%)$ & $58186(100 \%)$ & $118792(100 \%)$ \\
\hline
\end{tabular}

Fonte: Elaborado com base nos dados do Datasus (2021)

As internações por MAC geraram um custo total de 1.001.415.591,00 bilhões de reais em serviços hospitalares. O maior percentual de internações foi registrado no ano de 2019 com 11,93\% $(119.455 .795,19)$ dos custos totais. Na faixa etária menor que um ano houve maiores custos hospitalares (64,93\%; 650.210.624,70). A região com maior custo em internações foi a Sudeste $(45,45 \%$; 455.163.905,50) (Tabela 2).

Tabela 2 - Descrição dos custos em serviços hospitalares por internações de crianças com malformações congênitas do aparelho circulatório para as variáveis de ano de processamento, faixa etária e região de internação de 2010 a 2020.

\begin{tabular}{lc}
\hline \multicolumn{1}{c}{ Variáveis } & $\begin{array}{c}\text { Custo total } \mathbf{R} \$ \\
\mathbf{n}(\%)\end{array}$ \\
\hline $\begin{array}{l}\text { Custo por ano } \\
2010\end{array}$ & $67.229 .172,53(6,71 \%)$ \\
2011 & $79.133 .972,3(7,90 \%)$ \\
2012 & $76.796 .810,36(7,67 \%)$ \\
2013 & $81.932 .677,13(8,18 \%)$ \\
2014 & $85.076 .954,58(8,49 \%)$ \\
2015 & $86.749 .081,50(8,66 \%)$ \\
2016 & $84.997 .005,75(8,49 \%)$
\end{tabular}

\begin{tabular}{lr}
\hline \multicolumn{1}{c}{ Variáveis } & \multicolumn{1}{c}{$\begin{array}{c}\text { Custo total R\$ } \\
\mathbf{n}(\%)\end{array}$} \\
\hline 2017 & $93.922 .985,73(9,38 \%)$ \\
2018 & $113.208 .148,93(11,30 \%)$ \\
2019 & $119.455 .795,19(11,93 \%)$ \\
2020 & $112.944 .818 .05(11,29 \%)$ \\
Custo por Faixa etária & \\
$<1$ ano & $650.210 .624,70(64,93 \%)$ \\
1 a 4 anos & $257.335 .942,40(25,70 \%)$ \\
5 a 9 anos & $93.869 .024,16(9,37 \%)$ \\
Custo por Regiões de internação & \\
Sudeste & $455.163 .905,50(45,45 \%)$ \\
Nordeste & $209.779 .640,00(20,95 \%)$ \\
Sul & $189.565 .444,80(18,93 \%)$ \\
$\quad$ Norte & $64.234 .824,70(6,41 \%)$ \\
Centro-Oeste & $82.671 .776,22(8,26 \%)$ \\
Total & $1.001 .415 .591,00(100 \%)$ \\
\hline
\end{tabular}

Fonte: Elaborado com base nos dados do Datasus (2021)

Houve um total de 10.477 óbitos em crianças no período analisado (2010-2020), predominante no sexo masculino (52,63\%; 5514), com um maior percentual de óbito na região Sudeste, seguida da região Nordeste, com 37,73\% (3953/10477) e 26,98\% (2827/10477), respectivamente (Figura 2).

Figura 2 - Óbito por malformações congênitas do aparelho circulatório por região e sexo

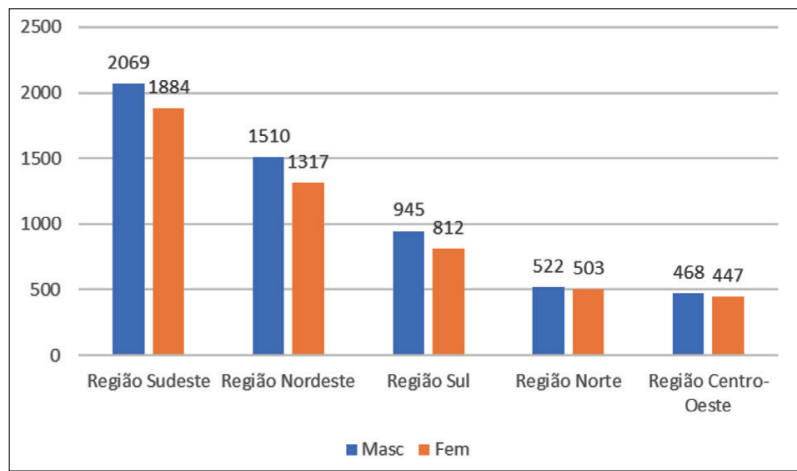

Fonte: Elaborado com base nos dados do Datasus (2021)

O maior número dos óbitos ocorreu em crianças abaixo de um ano $(85,85 \% ; 8995)$, sendo o sexo masculino nessa faixa etária o mais afetado com 53,07\% (4774) (Figura 3).

Figura 3 - Óbito por malformações congênitas do aparelho circulatório por faixa etária e sexo

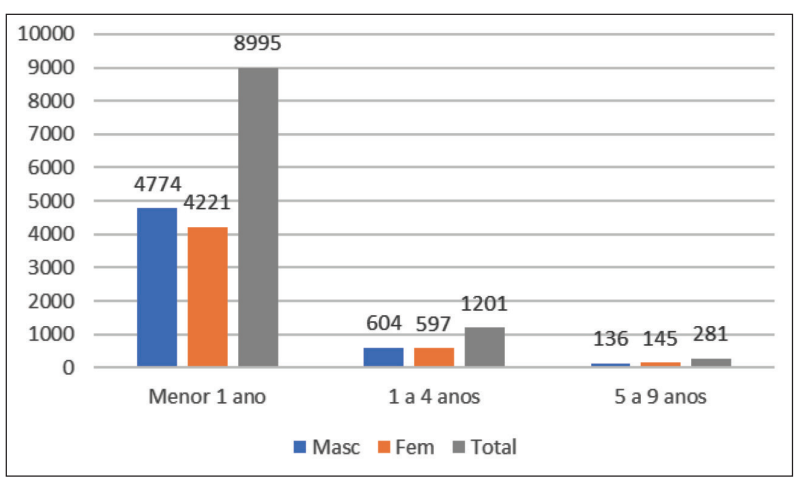

Fonte: Elaborado com base nos dados do Datasus (2021) 
Na evolução temporal dos óbitos ocorridos no período de 2010 a 2020, pode-se observar que 2017 foi o ano em que apresentou a maior frequência (9,62\%; 1008/10477), enquanto em 2013 houve o menor percentual de notificação de óbitos de crianças internadas com MAC no país $(8,39 \% ; 879 / 10477)$. A taxa de mortalidade total foi de $8,82 / 100$ habitantes, sendo maior no sexo masculino com $9,10 / 100$ mil habitantes (Figura 4).

Figura 4 - Óbito por MAC por ano de processamento e sexo

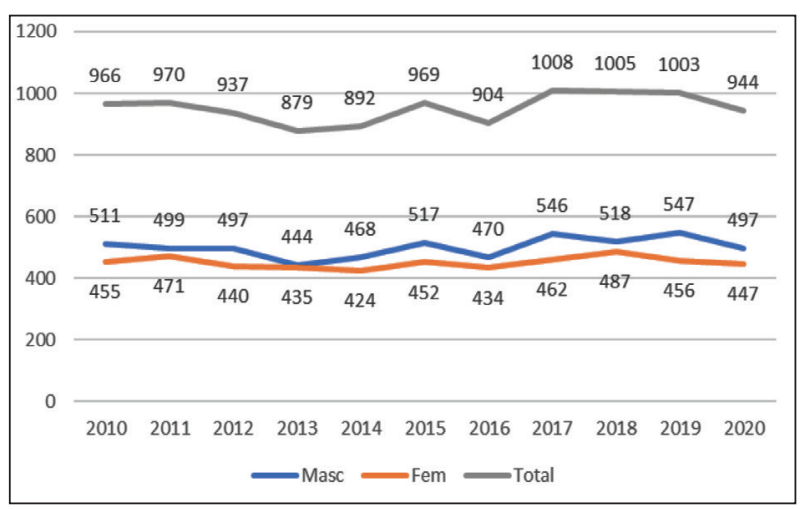

Nota: Taxa de mortalidade $-8,82 / 100$ habitantes (total); 9,10/100 mil habitantes (sexo masculino); 8,53/100 mil habitantes (feminino)

Fonte: Elaborado com base nos dados do Datasus (2021)

\section{DISCUSSÃO}

No Brasil, de 2010 a 2020 foram notificadas 118.792 internações por MAC, com um crescente aumento dos casos, entretanto, com pouca variação entre os anos, com queda das notificações no ano de 2020. Além disso, observou-se maior frequência de hospitalização, de custo dos serviços hospitalares e de óbitos em menores de um ano, sendo que a região Sudeste foi a que apresentou maior prevalência nas hospitalizações.

O declínio das internações notificadas nos anos 2019 a 2020 pode não refletir a real situação brasileira, tendo em vista que a pandemia do COVID-19 impôs o isolamento social, o receio da exposição em hospitais e consequente contaminação, além de uma diminuição no acompanhamento da mulher ao pré-natal (RAIMUNDO, 2021) e suspensão de internações eletivas durante o período. Houve ainda medo e ansiedade por parte dos genitores e de crianças em contrair a doença, gerando maior atraso no diagnóstico e maior progressão da enfermidade, repercutindo em um pior prognóstico (NORMANDO et al., 2021).

No mundo, estima-se que cerca de $6 \%$ dos nascidos vivos (NV) são diagnosticados com algum tipo de anomalia congênita, sendo estimado pela Organização Mundial de Saúde que cerca de 295 mil crianças morrem nas primeiras quatro semanas de vida (WHO, 2021). As anomalias congênitas representam atualmente a segunda principal causa de morte entre os menores de um ano no Brasil, segundo o Ministério da Saúde (2021), sendo as MAC as mais frequentes e com alta mortalidade (LANSKY; FRANÇA; LEAL, 2002; DATASUS, 2019). No entanto, no Brasil e em outros países da América Latina ainda há poucos trabalhos sobre as malformações cardíacas congênitas, principalmente devido ao fato que os estudos são realizados em centro de tratamento da doença, o que pode acarretar uma superestimação dos dados, não retratando sua real frequência na população (AMORIM et al., 2008; CATARINO et al., 2017).

Estudos mostram que das crianças que nascem com alguma MAC, sem intervenção clínica e cirúrgica, $14 \%$ não sobrevivem ao primeiro mês de vida e $30 \%$ ao primeiro ano de vida (BRUM; STEIN; PELLANDA, 2015). O Sistema de Informações sobre Nascidos Vivos (Sinasc), através da Declaração de Nascidos vivos (DNV), é a principal forma de captação das malformações congênitas no país (BRASIL. MINISTÉRIO DA SAÚDE, 2021).

Dentre as internações, 74.561 ocorreram em crianças abaixo de um ano, demonstrando a vulnerabilidade dessa faixa etária, gerando um custo significativo em serviços hospitalares. Cerca de 1 a 2 de cada mil nascidos vivos apresentam MAC grave, sendo que $30 \%$ recebem alta hospitalar sem diagnóstico preciso, podendo evoluir a óbito precoce antes de receber tratamento adequado (MELLANDER; SUNNEGARDH, 2006; WREN; REINHARDT; KHAWAJA, 2008).

Entretanto, medidas de promoção de saúde, como a realização do pré-natal e de ecocardiogramas fetais, poderiam reduzir o risco de óbito, possibilitando o diagnóstico precoce, conduta terapêutica adequada e encaminhamento desses pacientes para centros especializados, mesmo antes do nascimento (VICTORA et al., 2011), além de evitar internações recorrentes por complicações da cardiopatia, garantindo melhora na qualidade de vida (TRINDADE et al., 2010).

No período analisado, houve uma distribuição semelhante em relação ao sexo, sendo $51,02 \%$ do sexo masculino, corroborando outros estudos (MACIEL et al., 2006; RAMOS; CARVALHO; ZUGAIB, 2009). Neste estudo, houve o predomínio da cor branca $(35,99 \%)$, porém a cor não foi declarada em $29,65 \%$ dos casos.

Alguns estudos relataram que a frequência de alguns tipos de MC varia de acordo com a cor (BRASIL. MINISTÉRIO DA SAÚDE, 2021; CAPPELLESSO; AGUIAR, 2017; CHOU; CHAKRADHAR; GHIMIRE, 2019; HOLMES, 2006). Contudo, não há estudos conclusivos sobre a diferença racial na distribuição dos defeitos cardíacos, embora alguns estudos confirmem que os negros, quando comparados com brancos, têm maior incidência e cerca de $50 \%$ mais chances de as MAC serem mais graves e complexas (KNOWLES et al., 2017; MULLEN et al., 2021).

Um fator importante que talvez explique essas disparidades pode estar relacionado às variações genéticas subjacentes específicas dos grupos minoritários, entre outras causas potenciais, como fatores ambientais e condições socioeconômicas e sanitárias (CAPPELLESSO; AGUIAR, 2017; HOLMES, 2006; MULLEN et al., 2021).

O Sudeste foi a região que apresentou o maior percentual de internações e óbitos $(43,45 \%$ e $37,73 \%)$, seguida do Nordeste $(25,63 \%$ e $26,98 \%)$, correspondendo 
a $62,77 \%$ e $85,85 \%$ das internações e óbitos da população abaixo de um ano, respectivamente. Esse aumento no Sudeste pode ser explicado devido à maior concentração de pessoas nessa região. As diferenças entre acessibilidade geográfica, social, cultural e econômica do país aos recursos e ao acesso à saúde também são fatores relevantes (CANEO et al., 2012; DUNCAN et al., 2015; STOPA et al., 2017).

Outros estudos relatam que fatores de privação socioeconômica e o acesso reduzido a serviços de diagnóstico estão subjacentes às diferenças étnicas na MAC, em grupos minoritários (MULLEN et al., 2021).

A mortalidade infantil é um importante indicador de saúde, tendo em vista sua associação a fatores como saúde materna, qualidade e acesso a saúde pública, além de condições socioeconômicas, impactando em um diagnóstico precoce e melhor prognóstico (HOROVITZ et al., 2006). A falta de diagnóstico preciso das MAC, após o nascimento, é uma das possíveis causas para falhas na alimentação dos dados, levando a interpretações equivocadas, impactando negativamente no norteamento das condutas e gerando mais custos hospitalares (ANDRADE; ALVES; TORALLES, 2018).

Além disso, a internação de uma criança cardiopata, principalmente com a faixa etária menor que um ano, pode gerar custos mais elevados do que o habitual, devido à necessidade de cirurgias paliativas e/ou corretivas, além de maiores cuidados intensivos (PEIXOTO et al., 2011; ROSA et al., 2013), tanto que, neste estudo, $64,93 \%$ dos custos no período de 2010 a 2020 foram aplicados em crianças menores de um ano.

Este estudo apresenta como limitação a utilização de dados dependentes de registro prévio, podendo haver subnotificação dos casos de internação/óbitos por MAC identificados na fonte adotada.

\section{CONCLUSÃO}

No presente estudo, observou-se que as malformações congênitas do aparelho circulatório no período analisado apresentaram uma distribuição homogênea em relação ao sexo e número de internações de 2010 a 2020, entretanto, no ano de 2019 foi registrado o maior número de internações, com queda dos registros em 2020, o que pode estar relacionado à pandemia do COVID-19, a qual provocou isolamento social e receio quanto à procura das unidades hospitalares. Além disso, houve um maior número de internações da raça/cor branca, na região Sudeste e faixa etária menor que um ano, que gerou altos custos para os serviços hospitalares.

$\mathrm{O}$ avanço tecnológico possibilitou novos meios de diagnóstico pré-natal de anomalias fetais, como o principal método de rastreamento na população e, assim, promoveu uma redução dos índices de mortalidade. Medidas para um diagnóstico precoce devem ser realizadas para possibilitar a redução de internamentos, custos e óbitos nessa população.
Também é necessário avaliar as interrelações dos fatores maternos e socioeconômicos capazes de aumentar a prevalência de malformações do aparelho circulatório, por meio de políticas públicas que propiciem melhoria das condições de vida da população, a fim de reduzir a morbimortalidade infantil, de forma mais consistente e eficaz.

\section{REFERÊNCIAS}

AMORIM, L. F. et al. Presentation of congenital heart disease diagnosed at birth: analysis of 29,770 newborn infants. Jornal de Pediatria, Rio de Janeiro, v. 84, n. 1, p83-90, Jan-Feb. 2008.

ANDRADE, A. do N; ALVES, R. M.; TORALLES, M. B. P Perfil epidemiológico de anomalias congênitas. no Estado da Bahia. Revista de Ciências Médicas e Biológicas, Salvador, v. 17, n. 3, p. 287-291, 2018.

BRASIL. Ministério da Saúde. Secretaria de Vigilância em Saúde. Anomalias congênitas no Brasil, 2010 a 2019: análise de um grupo prioritário para a vigilância ao nascimento. Boletim Epidemiológico, Rio de Janeiro, v. 52, n. 6, 2021. Disponível em: https://www.gov.br/ saude/pt-br/media/pdf/2021/marco/3/boletim_epidemiologico_svs_6_ anomalias.pdf. Acesso em: 20 jul. 2021.

BRASIL. Ministério da Saúde. Portaria no 1.727, de 11 de julho de 2017. Aprova o Plano Nacional de Assistência à Criança com Cardiopatia Congênita. Diário Oficial [da] República Federativa do Brasil, Brasília, DF, n. 132, 12 de jul. 2017. Seção I, p. 47.

BRUM, C. D. A.; STEIN, A. T.; PELLANDA, L. C. Infant mortality in Novo Hamburgo: associated factors and cardiovascular causes. Arquivos Brasileiros de Cardiologia, São Paulo, v. 104, n. 4, p. 257-265, 2015.

CANEO, L. F. et al. A reflection on the performance of pediatric cardiac surgery in the State of São Paulo. Brazilian Journal of Cardiovascular Surgery, São Paulo, v. 27, n. 3, p. 457-462, 2012. DOI: 10.5935/16789741.20120076 .

CAPPELLESSO, V. R.; AGUIAR, A. P. Cardiopatias congênitas em crianças e adolescentes: caracterização clínico-epidemiológica em um hospital infantil de Manaus-AM. O Mundo da Saúde, [s.I.], v. 41, n. 2, p. 144153, 2017.

CATARINO, C. F et al. Registros de cardiopatia congênita em crianças menores de um ano nos sistemas de informações sobre nascimento, internação e óbito do estado do Rio de Janeiro, 2006-2010. Epidemiologia e Serviços de Saúde, Brasília, v. 26, p. 535-543, 2017.

CHOU, F-S.; CHAKRADHAR, R.; GHIMIRE, L. V. Socioeconomic and racial disparities in the prevalence of congenital heart disease in infants of diabetic mothers. The Journal of Maternal-Fetal \& Neonatal Medicine, [s.l.], n. 16, p. 1-4, 2019.

DATASUS. Ministério da Saúde. Brasília, 2021. Disponível em: https:// datasus.saude.gov.br/. Acesso em: 20 jun. 2021

DATASUS. Informações de saúde: morbidade e informações epidemiológicas. Brasília, 2019. Disponível em: www.datasus.gov.br. Acesso em: 28 jun. 2021.

DUNCAN, P. et al. "Health for All" in England and Brazil? International Journal of Health Services, Westport, v. 45, n. 3, p. 545-563, 2015. DOI: 10.1177/0020731415584558.

DUTRA, L. P.; LIMA, A. R. O.; PINTO JUNIOR, E. P. Características clínicas e epidemiológicas das malformações congênitas do sistema nervoso central em recém-nascidos. Journal of Chemical Information and Modeling, Washington, v. 110, n. 9, p. 1689-1699, 2017.

HOCKENBERRY, M. Fundamentos de enfermagem pediátrica. São Paulo: Elsevier, 2011. 
HOLMES, L. B. Congenital malformations. New England Journal of Medicine, Boston, v. 295, n. 4, p. 204-207, 2006.

HOROVITZ, D. D. G. et al. Atenção aos defeitos congênitos no Brasil: características do atendimento e propostas para formulação de políticas públicas em genética clínica. Cadernos de Saúde Pública, Rio de Janeiro, v. 22, p. 2599-2609, 2006.

KNOWLES, R. L. et al. Variação étnica e socioeconômica na incidência de cardiopatias congênitas. Archives of Diseases in Childhood, [s.l.], n. 102, p. 496-502, 2017. DOI: 10.1136/ archdischild-2016-311143.

LANSKY. S.; FRANÇA. E.; LEAL, M.C. Perinatal mortality and evitability: a review. Revista de Saúde Pública, Rio de Janeiro, v. 36, n. 6, p. 759772, 2002.

LEITE, D. de L.; MIZIARA, H.; VELOSO, M. Congenital cardiac malformations in pediatric necropsies: characteristics, associations and prevalence. Arquivos Brasileiros de Cardiologia, v. 94, p. 294-299, 2010.

MACIEL, E. L. N. et al. Perfil epidemiológico das malformações congênitas no município de Vitória-ES. Cadernos de Saúde Coletiva, Goiania, v. 14, n. 3, p. 507-518, 2006.

MARI, M.A. Avaliação do desenvolvimento infantil e a influência dos fatores biopsicossociais em crianças com cardiopatia congênita. 2015. Dissertação (Mestrado) - Universidade Federal do Rio Grande do Norte, Rio Grande do Norte, 2015.

MELLANDER, M.; SUNNEGARDH, J. Failure to diagnose critical heart malformations in newborns before discharge - an increasing problem? Acta Paediatrics. [s.I.], n. 95, p.407-413, 2006.

MIYAGUE, N. I. et al. Epidemiological study of congenital heart defects in children and adolescents: analysis of 4,538 cases. Arquivos Brasileiros de Cardiologia, São Paulo, v. 80, p. 274-278, 2003.

MULLEN, M. K. et al. Race and genetics in congenital heart disease: Application of iPSCs, Omics, and machine learning technologies. Frontiers in Cardiovascular Medicine, [s.I], v. 8, p. 37, 2021.

NORMANDO, P. G. et al. Redução na hospitalização e aumento na mortalidade por doenças cardiovasculares durante a pandemia da COVID-19 no Brasil. Arquivo Brasileiro de Cardiologia, São Paulo, v.116, n. 3, p. 371-380, 2021.

PEIXOTO, A. et al. Febre reumática: revisão sistemática. Revista da Sociedade Brasileira de Clínica Médica, São Paulo, v. 9, n. 3, p. 234238, 2011.
RAIMUNDO, V. K. Saúde reprodutiva na pandemia covid-19: os desafios e respostas na atenção ao pré-natal em Franco da Rocha. Physis: Revista de Saúde Coletiva, Rio de Janeiro, v. 30, n. 2, p. e300215, 2021.

RAMOS, J. L. de A. M; CARVALHO, M. H. B.; ZUGAIB, M. Caracterização sociodemográfica e resultados perinatais das gestações com diagnóstico ultrassonográfico de malformação fetal. Revista da Associação Médica Brasileira, São Paulo, v. 55, p. 447-451, 2009.

RICHMOND, S.; WREN, C. Early diagnosis of congenital heart disease. In: Seminars in neonatology. WB Saunders, [s.I.], v. 6, n. 1, p. 27-35, 2001.

RODRIGUES, L. S. et al. Características das crianças nascidas com malformações congênitas no município de São Luís, Maranhão, 20022011. Epidemiologia e Serviços de Saúde, Brasília, v. 23, n. 2, p.295304. 2014.

ROSA, R. C. M. et al. Cardiopatias congênitas e malformações extracardíacas. Revista Paulista de Pediatria, São Paulo, v. 31, p. 243251, 2013.

SANTOS, B. de G. M. et al. Correção cirúrgica de cardiopatias congênitas em recém-nascido. Insuficiência Cardíaca, Buenos Aires, v. 7, n. 4, p. 184-189, 2012.

SOARES, A. M. Mortalidade em Doenças Cardíacas Congênitas no Brasil-o que sabemos? Arquivos Brasileiros de Cardiololgia, São Paulo, v. 115, n. 6, p. 1174-1175, 2020.

STOPA S. R. et al. Use of and access to health services in Brazil, 2013 National Health Survey. Revista Saúde Pública, São Paulo, v. 51, supl. 1, 2017. DOI: 10.1590/s15188787.2017051000074.

TRINDADE, E. et al. Five-year follow-up of congenital heart surgery at the Heart of the Clinics Hospital. In: HTAi 2010-Maximising the Value of Health Technology Assessment. 6th $-9^{\text {th }}$. 2010. São Paulo: Medical School, 2010.

VICTORA C. G. et al. Saúde de mães e crianças no Brasil: progressos e desafios. Séries Saúde no Brasil 2. The Lancet.com, [s.I.], p. 32-46, 2011. Disponível em: https://edisciplinas.usp.br/pluginfile.php/156989/ mod_resource/content/1/Victora\%20saude $\% 20$ mulheres $\% 20 \mathrm{e} \% 20$ crian\%C3\%A7as\%20Lancet\%202011.pdf. Acesso em: 30 jun. 2021. DOI:10.1016/S01406736(11)60138-4.

WHO. Congenital anomalies. Genebra, 2020. Disponível em: https:// bit.ly/3aLluv1. Acesso em: 20 July 2021.

WREN, C.; REINHARDT, Z.; KHAWAJA, K. Twenty-year trends in diagnosis of life-threatening neonatal cardiovascular malformations. Arch Dis Child Fetal Neonatal, London, v. 98, n. 1, F33-F35, 2008.

Submetido em: 19/11/2021

Aceito em: 20/11/2021 